\title{
International Journal of Computer and Communication Technology
}

April 2014

\section{Spectral Minutiae Fingerprint Recognition System}

S. Shanawaz Basha

AVSV, Kurnool, AP, India,, shanawaz.basha@yahoo.com

N. Musrat Sultana

MGIT, Hyderabad, AP, India, sultanamgit@yahoo.com

Follow this and additional works at: https://www.interscience.in/ijcct

\section{Recommended Citation}

Basha, S. Shanawaz and Sultana, N. Musrat (2014) "Spectral Minutiae Fingerprint Recognition System," International Journal of Computer and Communication Technology. Vol. 5 : Iss. 2 , Article 6.

DOI: $10.47893 /$ IJCCT.2014.1228

Available at: https://www.interscience.in/ijcct/vol5/iss2/6

This Article is brought to you for free and open access by the Interscience Journals at Interscience Research Network. It has been accepted for inclusion in International Journal of Computer and Communication Technology by an authorized editor of Interscience Research Network. For more information, please contact sritampatnaik@gmail.com. 


\title{
Spectral Minutiae Fingerprint Recognition System
}

\author{
S.Shanawaz Basha ${ }^{1} \&$ N.Musrat Sultana ${ }^{2}$ \\ ${ }^{1}$ AVSV, Kurnool, AP, India, ${ }^{2}$ MGIT, Hyderabad, AP, India \\ E-mail : shanawaz.basha@yahoo.com, sultanamgit@yahoo.com
}

\begin{abstract}
Biometrics refers to the automatic recognition of individuals based on their physiological and/or behavioral characteristics, such as faces, finger prints, iris, and gait. In this paper, we focus on the application of finger print recognition system. The spectral minutiae fingerprint recognition is a method to represent a minutiae set as a fixedlength feature vector, which is invariant to translation, and in which rotation and scaling become translations, so that they can be easily compensated for. Based on the spectral minutiae features, this paper introduces two feature reduction algorithms: the Column Principal Component Analysis and the Line Discrete Fourier Transform feature reductions, which can efficiently compress the template size with a reduction rate of $94 \%$.With reduced features, we can also achieve a fast minutiae-based matching algorithm. This paper presents the performance of the spectral minutiae fingerprint recognition system, this fast operation renders our system suitable for a large-scale fingerprint identification system, thus significantly reducing the time to perform matching, especially in systems like, police patrolling, airports etc,. The spectral minutiae representation system tends to significantly reduce the false acceptance rate with a marginal increase in the false rejection rate.
\end{abstract}

Key words - Biometrics, fast minutiae matching, feature reduction, fingerprint identification, template protection.

\section{INTRODUCTION}

Fingerprints are fully formed at about seven months of fetus development. General characteristics of the fingerprint emerge as the skin on the fingertip begins to differentiate. Fingerprint recognition systems have the advantages of both ease of use and low cost. The Unisys Security Index released in December 2008 reveals that fingerprint is the most acceptable biometric technology [1]. Most fingerprint recognition systems are based on the use of a minutiae set. Minutiae are the endpoints and bifurcations of fingerprint ridges. They are known to remain unchanged over an individual's lifetime and allow a very discriminative classification of fingerprints [2].

Spectral minutiae representation presented in [3] is a method to represent a minutiae set as a fixed-length feature vector, which is invariant to translation, and in which rotation. And scaling become translations, so that they can be easily compensated for. These characteristics enable the combination of fingerprint recognition systems with recently developed template protection schemes based on fuzzy commitment and helper data schemes, such as [4] and [5], that require as an input a fixed-length feature vector representation of a biometric modality. The large dimensionality of the spectral minutiae feature can cause three problems.
First, the storage requirement for a spectral minutiae fingerprint system is very high.

In this paper, we will introduce two feature reduction methods in order to solve the above problems of the original spectral minutiae algorithm: the Column Principal Component Analysis (Column-PCA) and the Line Discrete Fourier Transform (Line-DFT) feature reduction algorithms. By applying Column-PCA and Line-DFT methods to the original spectral minutiae features, we can effectively compress the spectral minutiae templates and increase the matching speed.

For fingerprint identification systems with very large databases [8], in which a fast comparison algorithm is necessary, most minutiae-based matching algorithms will fail to meet the high speed requirement. Compared with other AFIS (Automated Fingerprint Identification System) vendors, our spectral minutiae fingerprint recognition system has the speed advantage: the experiment shows that our matching speed is more than 15 times higher than that of another commercial minutiae-based fingerprint matching algorithm (we will present the details later). To satisfy the high speed requirement, some AFIS vendors first use the global fingerprint characteristics (image-based features) as the first stage matching, and then use the minutiae matcher as the second stage matching [2]. The spectral minutiae

International Journal of Computer and Communication Technology (IJCCT), ISSN: 2231-0371, Vol-5, Iss-2 
representation we present this paper only needs the minutiae templates as input, so that it can be easily integrated into any arbitrary minutiae-based fingerprint recognition system. This motivates us to consider our spectral minutiae algorithm as a preselector (or prefilter) for a large-scale AFIS to improve the overall matching speed performance, especially in systems operating at geographical level (e.g., police patrolling) or in complex critical environments (e.g., airports). Besides the speed advantage, our algorithm can also be combined with template protection schemes, which gains more and more attention due to the substantial privacy concerns.

\section{SPECTRAL MINUTE REPRESENTATION}

The spectral minutiae representation is based on the shift, scale and rotation properties of the twodimensional continuous Fourier transform. In [3], the concept of two representation methods is introduced: the location-based spectral minutiae representation (SML) and the orientation-based spectral minutiae representation (SMO).

2.1. Location-Based spectral minutiae representation (SML)

When we are implementing the Fourier transform there are two important issues that should be considered. First, when a discrete Fourier transform is taken of an image, this results in a representation of a periodic repetition of the original image. This is undesirable because it introduces errors due to discontinuities at the image boundaries. Second, the re-mapping onto a polarlogarithmic coordinate system after using a discrete Therefore, we introduce an analytical representation of the input minutiae, and then use analytical expressions of a continuous Fourier transform that are evaluated on a grid in the polar-logarithmic plane. These analytical expressions are obtained as follows. Assume we have a fingerprint with $\mathrm{Z}$ minutiae. With every minutia, a function $m_{i}(x, y)=\delta\left(x-x_{i}, y-y_{i}\right), i=1, \ldots \ldots, Z$ is associated where $\left(x_{i}, y_{i}\right)$ represents the location of the $\mathrm{i}^{\text {th }}$ minutia in the fingerprint image. Thus the spatial domain, every minutia is represented by a Dirac pulse. The Fourier transform of $m_{i}(x, y)$ is given by

$$
\digamma\left\{\mathrm{m}_{\mathrm{i}}(\mathrm{x}, \mathrm{y})\right\}=\exp \left(-\mathrm{j}\left(\omega_{\mathrm{x}} x_{i}+\omega_{\mathrm{y}} y_{i}\right)\right)
$$

and the location-base spectral minutiae representation is defined as

$$
M_{L}\left(\omega_{\mathrm{x}}, \omega_{\mathrm{y}}\right)=\sum_{\mathrm{i}=1}^{\mathrm{z}} \exp \left(-\mathrm{j}\left(\omega_{\mathrm{x}} x_{i}+\omega_{\mathrm{y}} y_{i}\right)\right),
$$

We use a Gaussian low-pass filter to attenuate the higher frequencies, in order to reduce the sensitivity to small variations in minutiae locations in the spatial domain. This multiplication in the frequency domain corresponds to a convolution in the spatial domain where every minutia is now represented by a Gaussian pulse. Following the shift property of the Fourier transform, the magnitude of $M$ is taken in order to make the spectrum invariant to translation of the input and we obtain

$$
\begin{aligned}
& M_{L}\left(\omega_{\mathrm{x}}, \omega_{\mathrm{y}} ; \sigma_{\mathrm{L}}{ }^{2}\right) \\
& =\exp \left[-\frac{\omega_{\mathrm{x}}{ }^{2}+\omega_{\mathrm{y}}{ }^{2}}{2{\sigma_{\mathrm{L}}}^{-2}}\right] \sum_{\mathrm{i}=\mathbf{1}}^{\mathrm{z}} \exp \left(-\mathrm{j}\left(\omega_{\mathrm{x}} x_{i}+\omega_{\mathrm{y}} y_{i}\right)\right) \mid
\end{aligned}
$$

Equation (3) is the analytical expression for the spectrum which can directly be evaluated on a polarlogarithmic grid. The resulting representation in the polar-logarithmic domain is invariant to translation, while rotation and scaling of the input have become translations along the polar-logarithmic coordinates.

\subsection{Orientation-Based spectral minutiae representation} (SMO)

The location-based spectral minutiae representation (SML) only uses the minutiae location information. However, including the minutiae orientation as well may give better discrimination. Therefore, it can be beneficial to also include the orientation information in our spectral representation. The orientation $\boldsymbol{\theta}$ of a minutia can be incorporated by using the spatial derivative $m(x, y)$ of in the direction of the minutia orientation. Thus, to every minutia in a fingerprint, a function $m_{i}(x, y, \theta)$ is assigned being the derivative of $m_{i}(x, y)$ in the direction $\theta_{i}$, such that

$$
\begin{array}{r}
F\left\{\mathrm{~m}_{\mathrm{i}}(\mathrm{x}, \mathrm{y}, \theta)\right\}=\mathrm{j}\left(\omega_{\mathrm{x}} \cos \theta_{i}+\omega_{\mathrm{y}} \sin \theta_{i}\right) \\
. \exp \left(-\mathrm{j}\left(\omega_{\mathrm{x}} x_{i}+\omega_{\mathrm{y}} y_{i}\right)\right)
\end{array}
$$

As with the SML algorithm, using a Gaussian filter and taking the magnitude of the spectrum yields

$$
\begin{aligned}
& \left|M_{O}\left(\omega_{\mathrm{x}}, \omega_{\mathrm{y}} ; \sigma_{\mathrm{O}}{ }^{2}\right)\right|=\mid \exp \left[\frac{{\omega_{\mathrm{x}}{ }^{2}+\omega_{\mathrm{y}}{ }^{2}}_{2 \sigma_{\mathrm{O}}{ }^{-2}}}{\mathrm{z}}\right] \mathrm{X} \\
& \sum_{\mathrm{I}=\mathbf{1}} \mathrm{j}\left(\omega_{\mathrm{x}} \cos \theta_{i}+\omega_{\mathrm{y}} \sin \theta_{i}\right) \cdot \exp \left(-\mathrm{j}\left(\omega_{\mathrm{x}} x_{i}+\omega_{\mathrm{y}} y_{i}\right)\right) \mid
\end{aligned}
$$

\subsection{Implementation}

In order to obtain our final spectral representations, the continuous spectra (3) and (5) are sampled on a polar-logarithmic grid. In the radial direction $\lambda$, we use 
$M=128$ samples between $\lambda_{1}=0.1$ and $\lambda_{1}=0.6$. In the angular direction $\beta$, we use $N=256$ samples uniformly distributed between $\beta=0$ and $\beta=\pi$. Because of the symmetry of the Fourier transform for real-valued functions, using the interval between 0 and $\pi$ is sufficient. This polar-logarithmic sampling process is illustrated in Figs. 1 and 2.

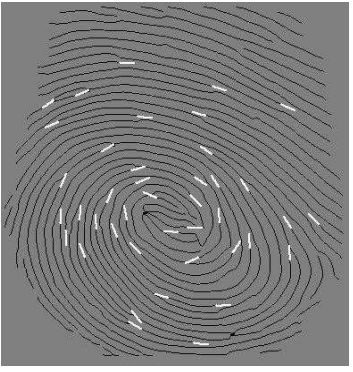

(1)

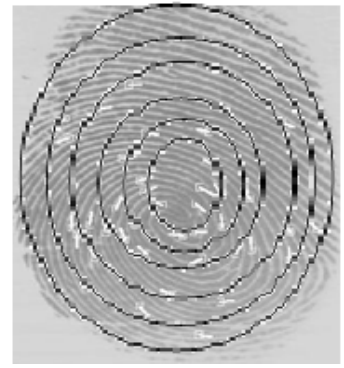

(2)
Fig. 1 : Illustration of polar logarithmic sampling (SML spectra). (1) Fourier spectrum in a Cartesian coordinate and a polar-logarithmic sampling grid. (2) Fourier spectrum sampled on a polar-logarithmic grid.

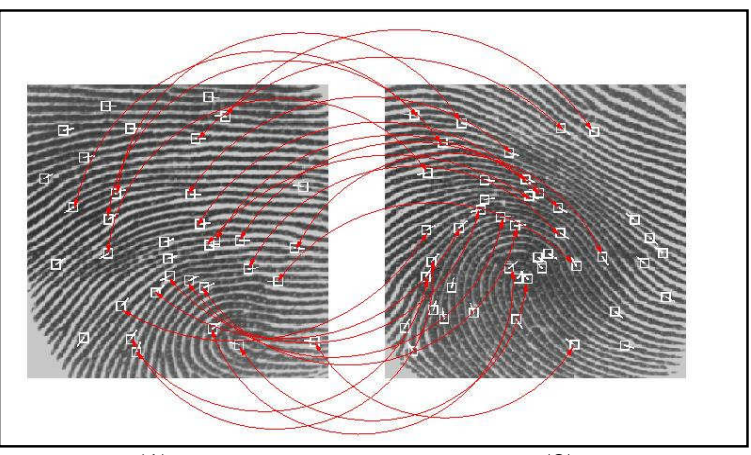

(1)

(2)

Fig. 2 : Illustration of the polar-logarithmic sampling (SMO spectra). (1)Fourier spectrum in a Cartesian coordinate and a polar-logarithmic sampling grid. (2) Fourier spectrum sampled on a polar-logarithmic grid.

The sampled spectra (3) and (5) will be denoted by $\mathrm{S}_{\mathrm{L}}\left(\mathrm{m}, \mathrm{n} ; \sigma_{\mathrm{L}}\right)$ and $\mathrm{S}_{\mathrm{O}}\left(\mathrm{m}, \mathrm{n} ; \sigma_{\mathrm{O}}\right)$ respectively, with $\mathrm{m}=$ $1, \ldots, \mathrm{M}, \mathrm{n}=1, \ldots . \mathrm{N}$. When no confusion can arise, the parameter $\sigma$ and the subscripts $\mathrm{L}$ and $\mathrm{O}$ will be omitted. For each spectrum, the horizontal axis represents the rotation angle of the spectral magnitude (from0 to $\pi$ ); the vertical axis represents the frequency of the spectral magnitude. It should be noted that the minutiae spectrum is periodic on the horizontal axis.

\subsection{Spectral minutiae matching}

Let $R(m, n)$ and $T(m, n)$ be the two sampled minutiae spectra respectively achieved from the reference fingerprint and the test fingerprint. Both $R$ $(m, n)$ and $T(m, n)$ are normalized to have zero mean and unit energy. We use the two-dimensional correlation coefficient between $R$ and $T$ as a measure of their similarity.

In practice, the input fingerprint images are rotated and might be scaled (for example, depending on the sensor that is used to acquire an image). Assume that the scaling has already been compensated for on the level of the minutiae sets [9]. Then we only need to test a few rotations, which become the circular shifts in the horizontal direction. We denote $T\left(m, n-n_{c s}\right)$ as a circular shifted version of $T(m, n)$. We use the fast rotation searching algorithm, based on variable step sizes that was presented in [10] and choose the maximum score of the different combinations as the final matching score between $R$ and $T$

$$
\begin{array}{r}
\mathrm{S}^{(\mathrm{R}, \mathrm{T})}=\max _{\mathrm{n}_{\mathrm{cs}}}\left\{1 / M N \sum \mathrm{R}(\mathrm{m}, \mathrm{n}) \mathrm{T}\left(\mathrm{m}, \mathrm{n}-\mathrm{n}_{\mathrm{cs}}\right)\right\}, \\
-15 \leq \mathrm{n}_{\mathrm{cs}} \leq 15 .
\end{array}
$$

\section{COLUMN-PCA FEATURE REDUCTION (CPCA)}

Spectral minutiae feature is a $d=M X N=32,768$ real-valued feature vector. This high dimensionality can cause the small sample size problem [7]. Small sample size effects are normally encountered in applications with high dimensional features and a complex classification rule, while the number of available training samples is inadequate. A mismatch between the actual statistics and the assumptions will lead to a loss of recognition performance. We can increase robustness against this type of mismatch by reducing the feature space dimension ability. PCA is a commonly used tool to achieve this.

\subsection{PCA Feature Reduction and Its Problem on Spectral Minutiae Representation}

In order to illustrate the problem of directly applying PCA on the features $S_{L}(m, n)$ and $S_{O}(m, n)$, let $\overline{0}$ $=\left(\mathrm{o}_{1}, \ldots \mathrm{O}_{\mathrm{D}}\right)^{\mathbf{T}}$ denote the unreduced spectral minutiae feature vector, that is, a one-dimensional form of the two- Dimensional spectral minutiae $S(m, n)$, with $m=$ $1, \ldots M n=1, \ldots N, \quad(M=128$ and $N=256)$. Thus, the dimensionality of $\overline{\mathrm{o}}$ is $D=M X N=32,768$.

If we have $L$ samples $\bar{o}_{1}, \ldots . . \overline{\mathrm{o}}_{\mathrm{L}}$ in the training set, we can create a $D X L$ data matrix $\mathbf{X}$ consisting of all the samples, as $\mathbf{X}=\left[\overline{\mathrm{o}}_{1}, \ldots \ldots \overline{\mathrm{o}}_{\mathrm{L}}\right]$. PCA can be implemented by doing singular value decomposition (SVD) on the matrix $\mathbf{X}$. 


$$
\mathbf{X}=\mathrm{UxSxVx}^{\mathrm{T}}
$$

with $\mathbf{U}_{\mathbf{X}}$ a $\mathrm{D} X \mathrm{X}(\mathrm{D}>\mathrm{L})$ orthonormal matrix spanning the column space of $\mathbf{X}, \mathbf{S}_{\mathrm{X}}$ a $L X L$ diagonal matrix of which the (non-negative) diagonal elements are the singular values of $\mathbf{X}$ in descending order, and $\mathbf{V}_{\mathbf{X}}$ a $L X$ $L$ orthonormal matrix spanning the row space of $\mathbf{X}$.

Let $\mathbf{U}_{\mathrm{X}}$ be the sub matrix of $\mathbf{U}_{\mathrm{X}}$ consisting of the first $D_{\mathrm{PCA}}\left(D_{\mathrm{PCA}}<L\right)$ columns, then we can implement PCA by

$$
\mathbf{Y}=\overline{\mathbf{U}}_{\mathbf{X}}^{\mathrm{T}} \mathbf{X}
$$

With $\mathbf{Y}$ the $D_{\mathrm{PCA}} X \quad L$ data matrix with reduced dimensionality. However, there are two problems in performing PCA to implement feature reduction on the minutiae spectra. The first is the small sample size problem [7]. In case the feature vector is $D=32,768$. An unreduced spectral minutiae representation, the dimensionality of the feature vector is . A reliable PCA feature reduction requires a large number of fingerprint samples to implement the PCA training, which is difficult to acquire. The second problem is that the minutiae spectra are not rotation-in-variant. As we mentioned in the previous section, the rotation of fingerprints becomes a circular shift of the minutiae spectra in the horizontal direction. For the PCA training, all the minutiae spectra must be aligned in order to get meaningful results. Then both the training and matching processes become complicated. To cope with the small sample size problem and to avoid the rotation alignment of minutiae spectra, we introduce the Column-PCA method to perform a feature reduction.

\subsection{Column-PCA: Feature Reduction without Small Sample Size Problems}

We first look at the spectral minutiae feature $S$ in the 2-D case as we presented in Section II-C. From Figs. 1 and 2 , we can see that the minutiae spectrum is periodic on the horizontal axis. Moreover, on the vertical axis, the spectra with different frequencies are correlated. Therefore, we consider using PCA to decorrelate the spectra with different frequencies in the vertical direction. To achieve this, we regard each column of $S$ as a new feature vector $\overline{\mathrm{e}}=\left(\mathrm{e}_{1} \ldots \mathrm{e}_{\mathrm{m}}\right)^{\mathrm{T}}$ then each (sampled) minutiae spectrum $\mathrm{S}(\mathrm{m}, \mathrm{n})$ consists of $N$ feature vectors $\overline{\mathrm{e}}, \mathrm{S}=\left(\overline{\mathrm{e}}_{1}, \ldots \overline{\mathrm{e}}_{\mathrm{n}}\right)$

If we have $L$ samples $S_{1} \ldots S_{L}$ in the training set, we can create a $\mathrm{M} \times \mathrm{L}_{\mathrm{N}}\left(\mathrm{L}_{\mathrm{N}}=\mathrm{N} \times \mathrm{L}, \mathrm{N}=256\right)$ data matrix $\mathbf{Z}$ consists of all the samples, as $\mathbf{Z}=\left[\mathrm{z}_{1}, . \mathrm{z}_{\mathrm{LN}}\right]$.

As we indicated in the previous section, another problem of directly using minutiae spectra to implement PCA feature reduction is that a rotation alignment of the minutiae spectra is needed, which is difficult to implement. In the spectral minutiae representation, the rotation operator commutes with column transformation. By using column feature vectors, the rotation variation becomes the samples sequence difference in the training procedure. This will not have any influence on the PCA feature reduction results. Therefore, by using column feature vectors to implement PCA feature reduction, we can cope with both the small sample size problem and avoid the rotation alignment of minutiae spectra as well. We call this method as the Column-PCA feature reduction (CPCA).

To implement CPCA, we first subtract the sample mean (column mean) from the data matrix $\mathbf{Z}$. The next step is to apply SVD on $\mathbf{Z}$

$$
\mathbf{Z}=\mathbf{U}_{\mathbf{Z}} \mathbf{S}_{\mathbf{Z}} \mathbf{V}_{\mathbf{Z}}^{\mathbf{T}}
$$

with $\mathbf{U}_{\mathbf{Z}}$ a $M X M$ orthonormal matrix spanning the column space of $\mathbf{Z}, \mathbf{S}_{\mathbf{Z}}$ a $M X M$ diagonal matrix of which the (non-negative) diagonal elements are the singular values of $\mathbf{Z}$ in descending order, and $\mathbf{V}_{\mathbf{Z}}$ a $L_{N} X$ $M$ orthonormal matrix spanning the row space of . Finally, we can obtain the CPCA transform matrix by retaining the first $M_{\mathrm{CPCA}}\left(M_{C P C A}<M\right)$ columns of $\mathbf{U}_{\mathbf{Z}}$ The CPCA transform on the minutiae spectra $S(m, n)$ is written as

$$
\mathbf{S}_{\mathrm{CPCA}}=\tilde{\mathrm{U}}^{\mathrm{T}}{ }_{\mathrm{Z}} \mathbf{S}
$$

With $\mathbf{S}_{\text {CPCA }}$ the $M_{\text {CPCA }} \mathrm{X} N$ data matrix with reduced dimensionality.

The CPCA transform is illustrated in Fig. 3(1) and 3(2) (here, we define $\tilde{\mathbf{U}}_{Z}^{*}=\mathbf{U}_{\mathrm{z}}$, that is $M_{C P C A}=M$, for a clear illustration). We can see that after the CPCA transform, the main energy of the original minutiae spectrum is concentrated in the top lines of $\mathbf{S}_{\text {CPCA. By }}$ only retaining the top $M_{C P C A}$ lines, we perform the CPCA feature reduction, with a reduction rate $\mathrm{R}_{\mathrm{CPA}}=(M$ - $\left.M_{C P C A}\right) / M$. Because the rotation operator commutes with column transformation, the minutiae spectrum $\mathbf{S}$ CPCA remains periodic on the horizontal axis after the CPCA transform

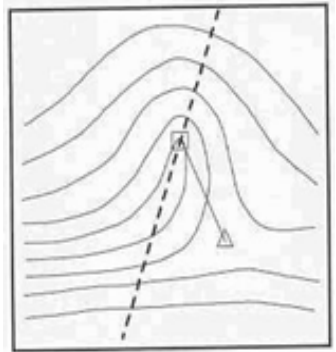

(1)

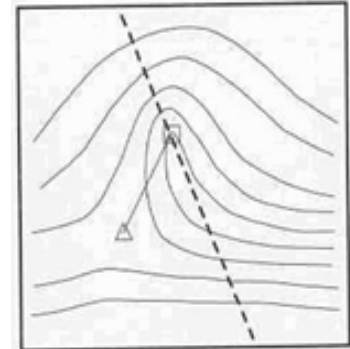

(2) 


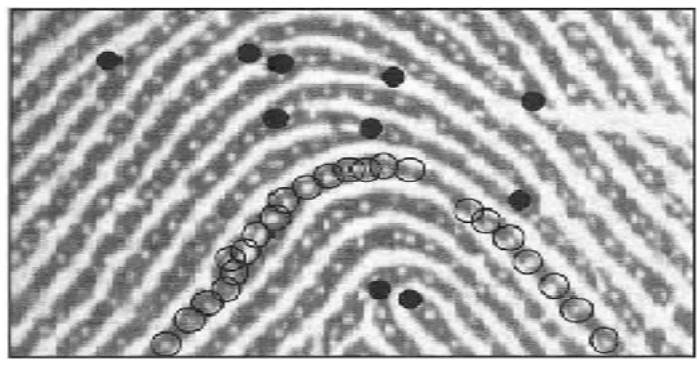

(3)

Fig. 3 : Illustration of the CPCA transform and the LDFT representation. (1) Location-based spectral minutiae. (2) Minutiae spectrum after the CPCA transforms. (3) Magnitude of the LDFT representation of (2).

\section{LINE -DFT FEATURE REDUCTION (LDFT)}

The CPCA feature reduction method reduces the minutiae spectrum feature $\mathbf{S}$ in the vertical direction. In this section, we will introduce the Line-DFT feature reduction (LDFT) method, which will reduce the feature in the horizontal direction. This method is based on the fact that the minutiae spectrum $\mathbf{S}$ is periodic on the horizontal axis. Therefore, it can be applied both independently and in combination with the CPCA feature reduction.

\subsection{Line-DFT Representation of the Minutiae Spectrum}

We denote each line of the minutiae spectrum $\mathbf{S}$ (here $\mathbf{S}$ can be the original minutiae spectrum or the minutiae spectrum after the CPCA feature reduction) as a line feature vector $\tilde{Y}=\left(\mathrm{y}_{1}, \ldots . \mathrm{y}_{\mathrm{n}}\right)$, thus $\mathbf{S}=\left(\tilde{\mathbf{y}}_{1}, \ldots\right.$. $\left.\tilde{\mathbf{y}}_{\mathbf{M}}\right)^{\mathbf{T}}$. Then we can regard each line feature vector as a periodic discrete-time signal (or sequence) with period $N$, and we denote this signal as $\mathrm{y}_{\mathrm{m}}(\mathrm{n})(m=1, \ldots \ldots M)(M$ $=128$ for the original minutiae spectrum or $M=M_{\mathrm{CPCA}}$ for the spectrum after the CPCA feature reduction). The discrete Fourier transform [12] of $y_{m}(n)$ is given by

$$
\begin{gathered}
\mathrm{Y}_{\mathrm{m}}(\mathrm{k})=\sum_{\mathrm{n}=0}^{N-1} \mathrm{y}_{\mathrm{m}}(\mathrm{n}) \exp (-\mathrm{j}(2 \pi / N) k n), \\
K=0,1 \ldots N-1, m=1, \ldots . M
\end{gathered}
$$

Because $y_{m}(n)$ is periodic, by performing DFT (implemented as FFT) on each line $y_{m}(n)$ of the minutiae spectrum $\mathbf{S}$, we can obtain, $\mathbf{S}_{\mathbf{L D F T}}=\left(\mathrm{Y}_{1}(\mathrm{k})\right)^{\mathrm{T}}$, $\mathbf{S}_{\text {LDFT }} \dot{\varepsilon} \mathrm{C}^{M}$ which is an exact representation of $\mathbf{S}$.

\subsection{Transform of Fourier Components to a Real-Valued} Feature Vector

Consider two discrete-time, periodic signals $f_{1}[n]$ and $f_{2}[n], f_{1}[n], f_{2}[n] \dot{\varepsilon} \mathrm{R}^{\mathrm{N}}$ with period $N(N$ is an even number), and their discrete Fourier transform are
$\mathrm{F}_{1}[\mathrm{k}]$ and $\mathrm{F}_{2}[\mathrm{k}]$ respectively. The DFT is orthnormal, thus it preserves inner products. Therefore, because of the symmetry properties of the DFT for real-valued signals, the correlation of $f_{1}[n]$ and $f_{2}[n]$ becomes

$$
\underset{\mathrm{n}=0}{\sum f_{1}[n] f_{2}[n]=1 / N\left(\mathrm{~F}_{1}[0] \mathrm{F} 2[0]+2 \mathrm{R} \sum_{k=1}^{N / 2} \mathrm{~F}_{1}[\mathrm{k}] \mathrm{F}_{2}[\mathrm{k}]\right)}
$$

Therefore, we can generate two one dimensional real-valued feature vectors $\mathbf{v}_{\mathbf{1}}$ and $\mathbf{v}_{\mathbf{2}}$ from the Fourier components.

\section{EXPERIMENTS}

\subsection{Measurements}

We test the spectral minutiae representation in a verification setting. The matching performance of a fingerprint verification system can be evaluated by means of several measures. Commonly used are the false acceptance rate (FAR), the false rejection rate (FRR), and the equal error rate (EER). In this paper, we use FAR, EER and the genuine accept rate (GAR), GAR = 1- FRR, as performance indicators of our scheme.

\subsection{Results without CPCA and LDFT Feature Reductions}

For a comparison with the results after the CPCA and the LDFT feature reductions, we first tested our algorithm without feature reductions. The results are shown in Table I and the ROC curves are shown in Figs. 4(a) and 5(a). From the results, we can see that the MCYT database received much better results than the FVC database. This shows that our algorithms are sensitive to the minutiae quality and fingerprint quality.

Table 1

Results without CPCA and LDFT feature reductions

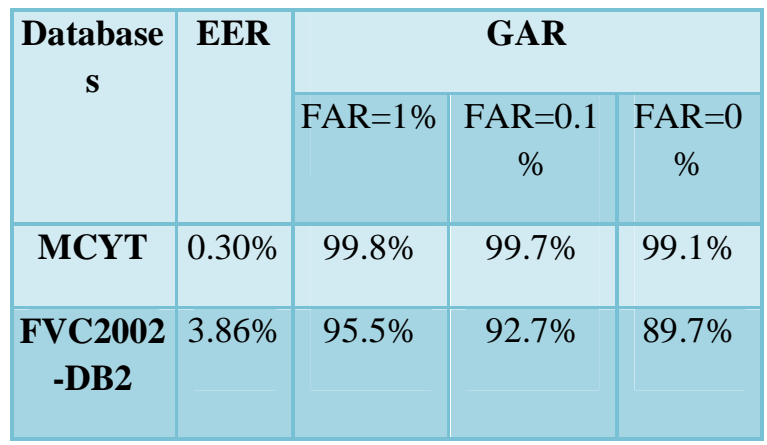

5.3. Results after CPCA and LDFT Feature Reductions

In case of using SML and SMO fusion, the spectral 
minutiae representation results in a 65536 real-valued feature vector. For fingerprint identification systems with very large databases, using the spectral minutiae representation requires a big template storage space and its matching speed is also limited. Therefore, applying the proposed CPCA and LDFT feature reduction algorithms is needed.

Table 2

Results after the CPCA feature reduction

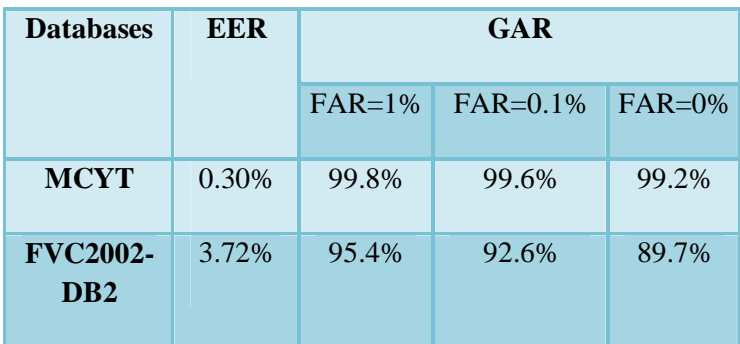

Table 3

Results after the LDFT feature reduction

\begin{tabular}{|c|c|c|c|c|}
\hline Databases & EER & \multicolumn{3}{|c|}{ GAR } \\
\cline { 3 - 5 } & & FAR=1\% & FAR=0.1\% & FAR=0\% \\
\hline MCYT & $0.29 \%$ & $99.8 \%$ & $99.7 \%$ & $99.1 \%$ \\
\hline $\begin{array}{c}\text { FVC2002- } \\
\text { DB2 }\end{array}$ & $3.72 \%$ & $95.6 \%$ & $92.7 \%$ & $89.7 \%$ \\
\hline
\end{tabular}

\subsection{Comparison}

We compared our results with other well-known minutiae matchers on the FVC2002-DB2 database: VeriFinger and Fuzzy Vault according to the protocol. We notice that the commercial minutiae matcher VeriFinger received much better results than ours.

However, the spectral minutiae algorithm is not robust to the low quality fingerprints. The fingerprint outliers will degrade the recognition accuracy, which limits the application of the spectral minutiae algorithm.

\section{CONCLUSIONS}

The experiments show that the Column-PCA and the Line-DFT feature reduction algorithms methods effectively decrease the spectral minutiae feature dimensionality with a reduction rate of $94 \%$, while at the same time, the recognition performance of the fingerprint system is not degraded. The proposed spectral minutiae fingerprint recognition system also shows a promising matching speed with 125000 comparisons per second. This algorithm overcomes the speed disadvantage of most minutiae-based algorithms and enables the application of a minutiae-based fingerprint identification system with a large database.

In this paper, we present the experimental results using two fingerprint databases: the MCYT and the FVC2002-DB2 databases. The MCYT database gives much better results than the FVC database. This shows that our algorithms are sensitive to the minutiae quality as well as the fingerprint quality.

\section{REFERENCES}

[1] Spotlight on Biometrics [Online]. Available: http://www.unisyssecuri-tyindex.com//

[2] D. Maltoni, D. Maio, A. Jain, and S. Prabhakar, Handbook of Finger print Recognition. New York: Springer, 2003.

[3] H. Xu, R. Veldhuis, A. Bazen, T. Kevenaar, T. Akkermans, and B. Gok-berk, "Fingerprint verification using spectral minutiae representations," IEEE Trans. Inf. Forensics Security, vol. 4, no. 3, pp. 397-409, Sep.2009.

[4] P. Tuyls, A. Akkermans, T. Kevenaar, G. Schrijen, A. Bazen, and R.Veldhuis, "Practical biometric authentication with template protection," in Proc. AVBPA, 2005.

[5] A. Juels, "Fuzzy commitment," in Security With Noisy Data-On Private Biometrics, Secure Key Storage and Anti-Counterfeiting, P. Tuyls B. Skoric, and T. Kevenaar, Eds. : Springer, 2007, ch. 3, pp. 45-56.

[6] K. Nandakumar, A. Jain, and S. Pankanti, "Fingerprint-based fuzzy vault: Implementation and performance," IEEE Trans. Inf. Forensics Security, vol. 2, no. 4, pp. 744-757, Dec. 2007.

[7] S. Raudys and A. Jain, Small Sample Size Effects in Statistical Pat tern Recognition: Recommendations for Practitioners vol. 13, no. 3, pp. 252-264, Mar. 1991.

[8] U.S. Visitor and Immigrant Status Indicator Technology Program (US-VISIT) [Online]. Available: http://www.dhs.gov/us-visit 